\title{
Clinical supervision
}

\author{
Duncan McLean
}

Most trainers and trainees in psychiatry would subscribe to the importance of clinical supervision. This is not borne out by the numbers of references to be found on the subject, especially on this side of the Atlantic. In America the journal Clinical Supervisor is dedicated to this topic. However, even there most authors come from disciplines other than psychiatry such as psychology, social work and psychotherapy. This relattve lack of interest in clinical supervision may be a by-product of our system of psychiatric training in which trainees rotate rapidly through a series of posts, so that despite the system of clinical tutors no one individual bears responsibility for a trainee's professional development. In the UK, authors on the subject tend to be current or recent trainees, and their papers often attest to the importance that trainees place on clinical supervision. However, once training is completed, interest in the topic appears to decline rapidly.

It is apparent that clinical supervision goes beyond the teaching of factual information, though one aspect must be to teach the application of empirical findings within the clinical situation. This means both the interpretation of research findings as well as use of the scientific method; that is of theory formation, hypothesis testing, and application to clinical problems. It has been suggested that clinical supervision itself should also be treated in a scientific fashion. Academic study leading to empirical trials could discover what is best both in content and method for clinical supervision. There is no doubt that academic interest and empirical testing would strengthen critical thinking about clinical supervision and possibly answer some questions about how it is best conducted, for example is supervision best done as an inter- or intra-disciplinary exercise.

However, clinical decision making cannot rely solely on scientific principles. Judgements and decisions have to be arrived at within the context of a professional relationship. Each clinical situation is unique. Although a professional may use the best available evidence, there are necessarily areas of doubt so that ethical, social and philosophical considerations will inevitably play a part in the outcome of a clinical encounter.

Clinical supervision therefore includes the induction into a professional Weltanschauung. This social process should not be underestimated, though it is usually only in retrospect that one can see the extent to which an individual's practice is embedded in a particular cultural view. Nevertheless, becoming part of a professional community is enhancing to the trainee. At the very least it offers a bedrock of ethical practice that both supports the professional in making difficult decisions as well as protecting the patient from the wilder vagaries of the clinicians' personal proclivities.

Within a broadly accepted framework of ethical practice there is room for considerable difference of opinion. Good clinical supervision should consider these issues as an aid to critical thinking. Some situations readily lend themselves to this, such as some borderline decisions about sectioning patients under the Mental Health Act. However, all clinical decisions rest within an ethical framework, and a trainee's ability to grasp this and face the inevitable anxieties and uncertainties of reaching a personal stance is the essence of becoming a professional.

A further issue in clinical supervision is considering the quality of the relationship between professional and patient. From persuading a patient that admission is in their best interest to the timing and tact of an interpretation in dynamic psychotherapy, the clinical aim can either founder or succeed. Some people believe such interpersonal expertise cannot be taught and that only exposure, and the trainee's trial and error within the clinical situation will gradually produce a personally effective style. On the other hand some clinical supervisors see this as the central aim of supervision and the exploration of the trainee's personal development as a legitimate area for consideration. In its most extreme form, as practised by some in America, there is little difference between supervision and personal therapy. Such a blurring of boundaries is unlikely to find much favour here. However, a discussion about the general characteristics of the interaction between a trainee and patient without going into the trainee's personal history or ways of relating can be llluminating and enable more clear sighted decision-making. For example. recognising a patient's subtly provocative behaviour and its likelihood of arousing anger in the trainee. If personal issues seriously interfere with a trainee's professional ability then this is not a matter for supervision, but should be dealt with 
through the clinical tutor and management structures.

It is not surprising that the variety of philosophical and scientific divergencies in psychiatry are reflected in the wide variety of clinical supervision methods. It is interesting to note in reading accounts of supervision how it mirrors the clinical method. Cognitive therapists will have clear and specific objectives in supervision, while dynamic supervision tends to be a reflective exploration of countertransference issues. Modelling can be an effective means of learning, but this parallel process in the supervision also demonstrates the blas of the supervisor. As such, the diversity of forms of clinical supervision in the Health Service is to be welcomed, but there should be greater academic interest to promote critical thinking about the scientific, ethical, and relational aspects of clinical supervision.

There is also a need for a more direct stimulus to quality in clinical supervision. Educationalists have developed a concept of reflective practice in relation to teaching methods which eschews any prescriptive or rigid adherence to a particular method, but encourages educational establishments to set up systems for continuous evaluation of practice. This approach can encourage innovation, and challenge narrow or ineffective methods. Such evaluation is rarely a part of clinical supervision. Although supervisors may not always welcome such methods that include having feedback about their performance from trainees, there is little doubt that this and other methods of evaluation would enhance performance.

Duncan McLean, Consultant Psychotherapist, King's College Hospital and The Anna Freud Centre

Correspondence: The Anna Freud Centre, 21 Maresfield Gardens, Hampstead, London NW3 5SH

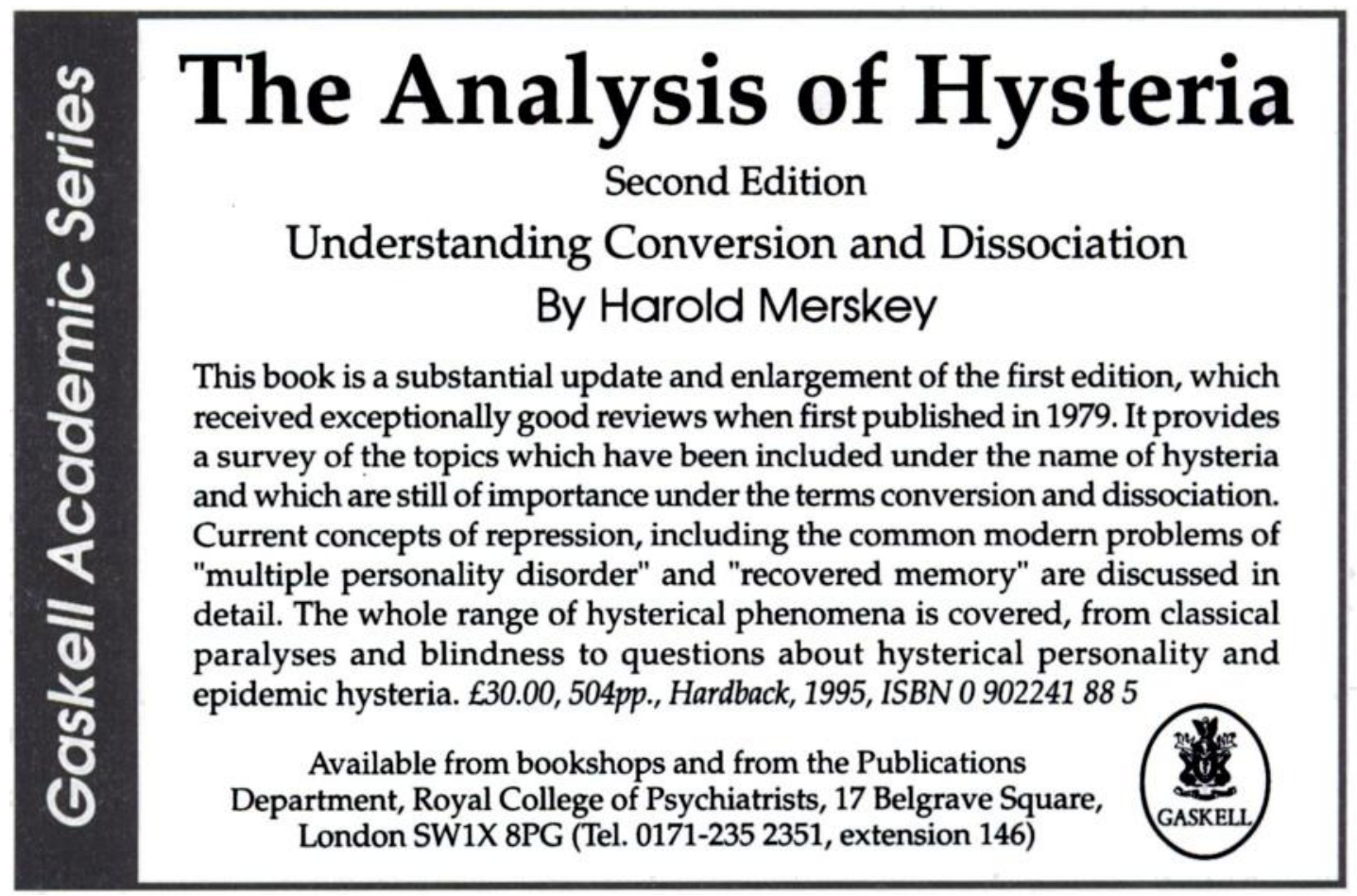

